\title{
STUDIES IN A CASE OF 'RENAL RICKETS'
}

BY

\author{
KENNETH SINCLAIR \\ From the Royal Infirmary, Edinburgh
}

(RECEIVED FOR PUBLICATION NOVEMBER 27, 1955)

The syndrome of secondary hyperparathyroidism due to chronic renal insufficiency is well recognized. A full discussion of the mechanisms involved can be found in Albright and Reifenstein's book (1948). Briefly, the sequence of events is renal insufficiency, phosphorus retention, a low serum calcium level and resulting parathyroid hyperplasia with skeletal changes and frequently metastatic calcification.

The condition can occur in both acquired and congenital renal disease with both glomeruli and tubules, or tubules alone, affected. The skeletal changes associated with the Fanconi syndrome (1936) are an example of the latter, which is discussed by McCune, Mason and Clarke (1943) in their paper. In this type phosphate is not retained.

Hyperparathyroidism secondary to renal disease can occur at any age. As a rule the renal failure is of considerable duration. Some of the changes described by Ellis and Evans (1933) in a series of children seem due to this condition. The skeletal changes in children are described in detail by Gilmour (1947).

The following case is recorded, first, because unusually severe skeletal changes were the presenting features in an illness which investigation showed to be renal in origin, although symptoms of renal failure were initially minimal; secondly, the results of full investigation were available for study.

\section{Case Report}

The patient was a girl aged 16 years. She was referred to the neurological clinic in May, 1954, and admitted directly to the ward. She had complained of pain in both knee caps for one year. Her parents thought she had been rather pale for a longer period. In August, 1953, she was seen to be dragging the right leg towards the end of the day. By February, 1954, she had developed a knock-knee deformity of both legs. From then until she was admitted to hospital her walking had become steadily worse with a scissor type of gait. She complained of some breathlessness on exertion. She had no frequency, nocturia or other urinary symptom.
Menstruation had begun at 14 years and was normal, $3 / 28$ days. There was no known previous renal disease or streptococcal infection. Her mother and father were well. Her brother was fit, being an Army cadet at Sandhurst. One cousin was a mental defective and epileptic.

On examination the patient was small for her age and pale, but the secondary sex characters were well developed. A dorsal kyphoscoliosis was present, the radii and ulnae were bowed, the costochondral junctions were much enlarged visibly and palpably. The lower limbs showed severe genu valgum with slight pes cavus. The pulse was $120 / \mathrm{min}$. regular. Blood pressure was $130 / 80 \mathrm{~mm}$. Hg. The heart was not clinically enlarged. A systolic murmur was heard over the apex and pulmonary areas. There was no oedema. The respiratory system was normal. The liver, sploen and kidneys were not palpable. The central nervous system and fundi were normal; particularly no neurological signs were found in the lower limbs, where power and coordination were normal considering the bony deformity. No enlarged lymph node or other swelling was palpable in the neck.

Investigations on May 22 showed the urine to be pale and clear with a specific gravity of 1,010 , an alkaline reaction and albumin, 2 g. 1 . In a centrifuged deposit, an occasional hyaline cast was found. The urine was sterile on culture and cloudy on the Sulkowitch test.

The urinary findings showed little variation during the patient's stay in hospital.

On May 23 the blood E.S.R. was $40 \mathrm{~mm}$. in the first hour (Westergren), Hb $40 \%$ (Sahli), R.B.C. $1.94 \mathrm{~m}$. c.mm., W.B.C. 4,100/c.mm. The Wassermann and Kahn reactions were negative. A film showed microcytic and hypochromic red blood cells and normal white blood cells.

Radiographs taken on May 26 showed that both kidneys were exceedingly small, with no definite renal calcification but calcification of pelvic vessels. The skeletal changes are shown in Figs. 1-5.

Because of the patient's poor general condition and renal function, neither intravenous nor retrograde pyelography was carried out.

The patient showed no change while she was in hospital. Daily urine examination showed the specific 


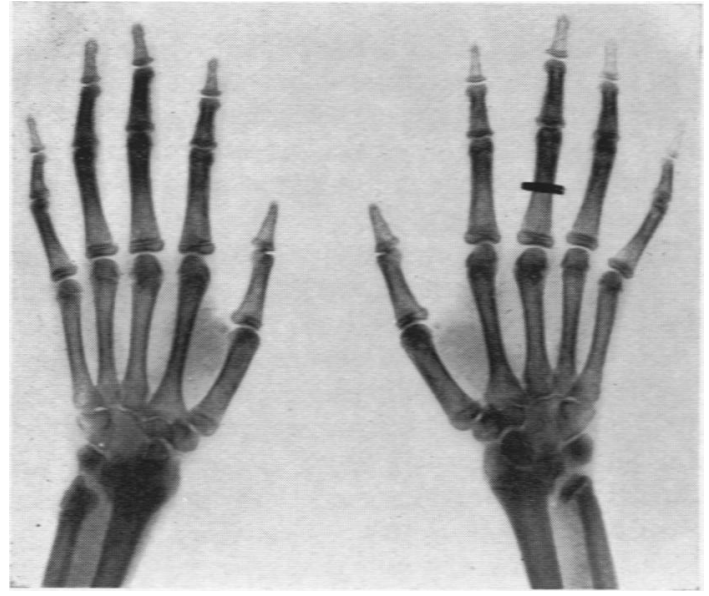

FrG. 1

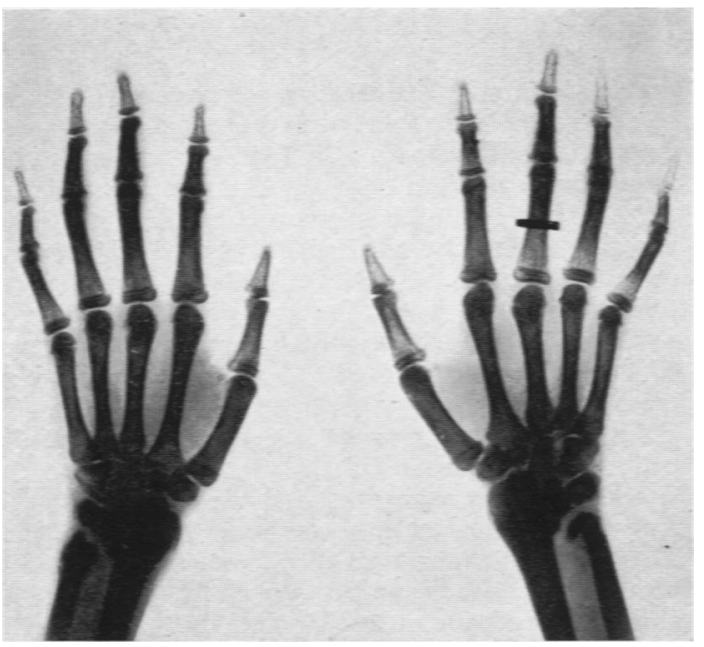

Frg. 2

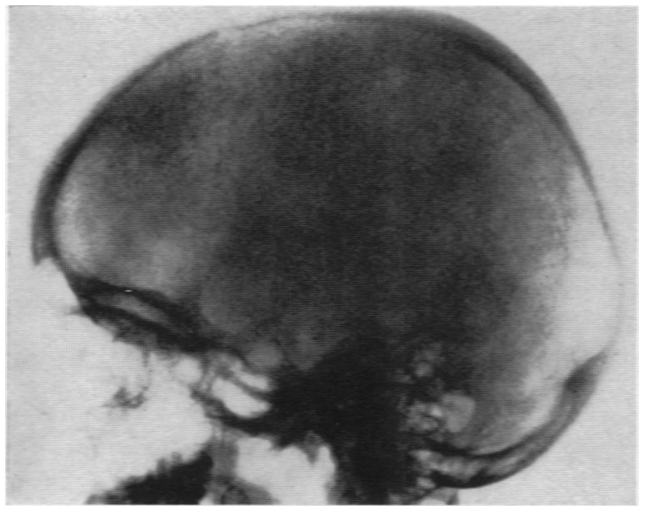

Fig. 3

New bone formation was seen at all costochondral regions, and faintly calcified osteoid at the outer ends of the clavicles. The upper ends of the humeri showed demineralization and deformity with the 'rotting stump' appearance. There was subluxation of epiphyses at the wrists. There was lace-like periosteal new bone formation in the hands, radii and ulnae. The skull showed pepper-pot demineralization. The legs showed genu valgum with changes in the metaphyses. The popliteal vessels were cakified.
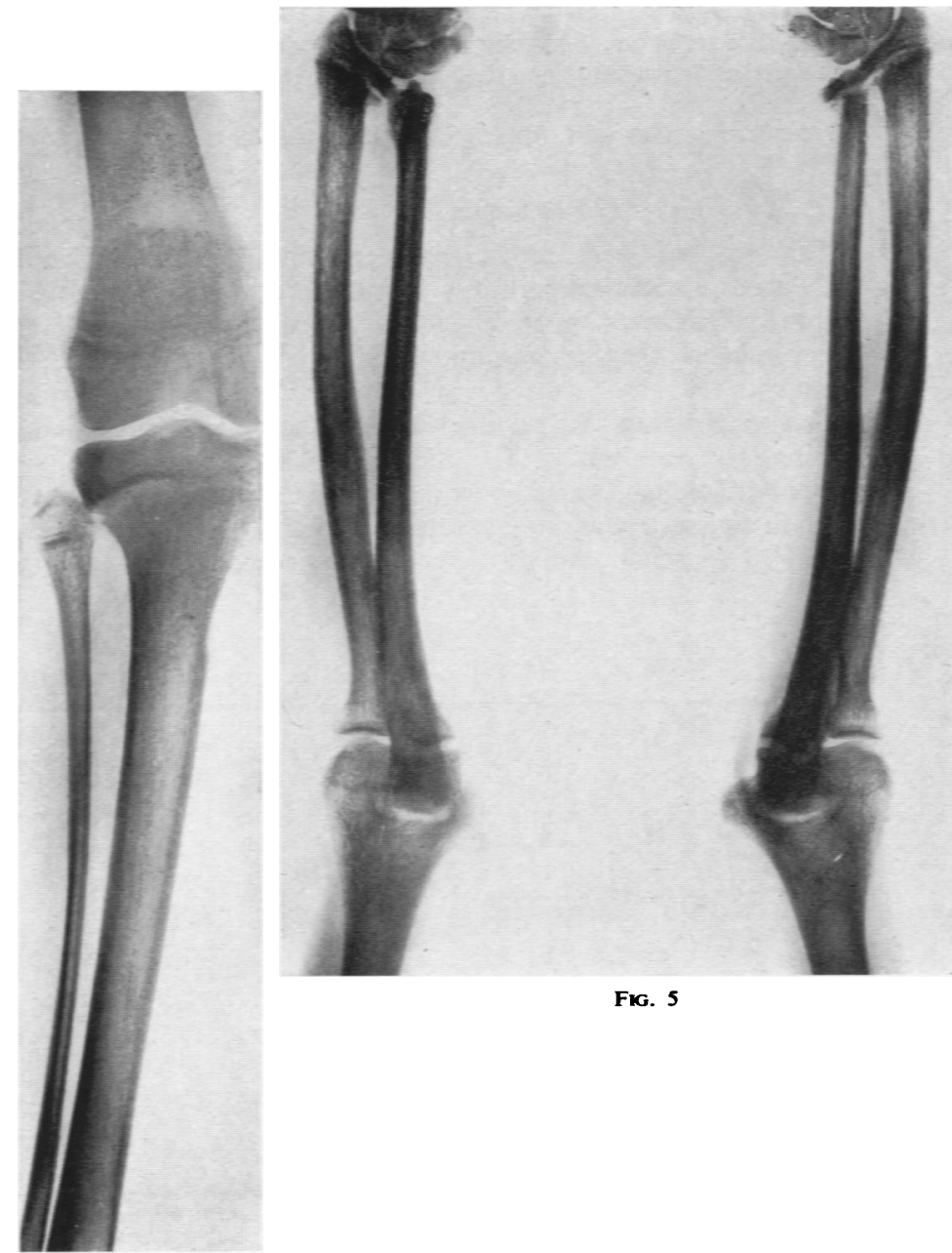

Fig. 5

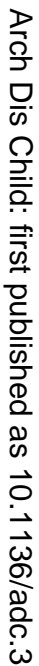

$\omega$

ज़

뭉

으

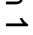

$\frac{1}{0}$

․ㅡ.

它

ภ ศి

잉

$\lesseqgtr$.

을

西

응

$\stackrel{\mathbb{1}}{2}$

$\overrightarrow{\overrightarrow{0}}$

3

항

ڤั)

웅

8

3

을.

N

$\sigma$

N 
gravity fixed at 1,010 , reaction alkaline and $1 \cdot 5-2 \mathrm{~g} . / 1$. of albumin. There were never more than a few hyaline casts in a spun deposit. On June 10, 3 pints of blood were given, and the haemoglobin rose from $40 \%$ to $57 \%$. The level after further transfusion on July 2 was $65 \%$. She also received $600 \mathrm{mg}$. \% of 'ferrivenin' in divided doses, but with little effect. From June 26 onwards she was given 30 grains pot. cit. four-hourly. On June 28 the $\mathrm{CO}_{2}$-combining power had riven to $63 \mathrm{vol}, \%$, but fell to $34 \mathrm{vol} . \%$ on June 30 . The blood pressure varied from $150 / 100$ to $130 / 80 \mathrm{~mm}$./ Hg. The E.S.R. varied from 95 to $40 \mathrm{~mm}$. in the first hour. The patient went home on July 3.

Following an epistaxis she was readmitted on August 26. Her general condition had deteriorated. She was very ill, breathless at rest and pale, with oedema of the face. Haemoglobin was $25 \%$, pulse 120 per minute, blood pressure $140 / 90 \mathrm{~mm}$./Hg. On August 27 the urine was unchanged except that it contained $3 \mathrm{~g}$./1. albumin. The reaction was alkaline (for biochemical findings see Table 1).

The patient was given 4 pints of blood and the haemoglobin rose to $70 \%$. She returned home on August 28. She deteriorated steadily and died at home in November, 1954.

No necropsy was performed.

\section{Discussion}

This patient had severe hyperparathyroidism with gross skeletal deformity secondary to renal failure which was probably due to congenital hypoplasia of the kidneys and was the cause of death.

The renal lesion was relatively silent, the patient coming to hospital with difficulty in walking, and painful and deformed lower limbs. On investigation, the picture was in fact one of severe 'renal rickets', a renal osteitis fibrosa generalisata.
Albright, Drake and Sulkowitch (1937) suggest that this condition resembles true rickets only in the radiological appearances. At the same time, they point out that epiphyseal changes are never seen in primary hyperparathyroidism without accompanying renal failure.

The radiological evidence for hyperparathyroidism in this case is extensive, e.g., the pepper-pot skull, the fine periosteal new bone formation and the arterial calcification in someone aged 16 years. (The teeth were not radiographed but in less obvious cases the absence of the lamina dura around them may be helpful (Strock, 1941).) These changes are superimposed on widespread faulty calcification of osteoid as seen in rickets.

The biochemical findings of normal low serum calcium, high serum inorganic phosphorus, high alkaline phosphatase plus the serum and urine calcium levels on a low calcium intake are in keeping with hyperparathyroidism.

That the renal failure was severe is shown by the low fixed specific gravity of the urine, the poor urea clearance, the raised serum phosphorus, creatinine and non-protein nitrogen, plus the low $\mathrm{CO}_{2}$ combining power. A congenital cause for this is suggested by the absence of any acute episode in a child, and the paucity of uraemic symptoms in the face of such a degree of renal failure. The extremely small renal shadows also favour congenital hypoplasia.

It may be that a few years before coming to hospital the patient and her metabolism outgrew the functional capacity of the kidneys with ensuing renal failure and hyperparathyroidism. Severe renal damage may be the presenting feature in primary hyperparathyroidism, but the absence of

TABLE 1

BIOCHEMICAL FINDINGS

\begin{tabular}{|c|c|c|c|c|c|c|c|c|c|c|c|c|c|c|c|}
\hline & $\frac{\frac{\hbar}{n}}{\frac{n}{d}}$ & $\frac{\pi}{\frac{n}{n}}$ & 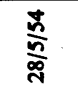 & $\frac{\frac{\pi}{n}}{\frac{\pi}{d}}$ & $\frac{\hbar}{\frac{n}{d}}$ & $\frac{\frac{5}{n}}{\frac{n}{m}}$ & $\frac{\frac{\pi}{0}}{n}$ & $\frac{\frac{\hbar}{0}}{\frac{\partial}{\gamma}}$ & $\frac{\frac{1}{\sigma}}{\frac{\sigma}{\sigma}}$ & $\frac{\frac{\hbar}{\sigma}}{a}$ & $\frac{\frac{\hbar}{0}}{0}$ & $\frac{\frac{\hbar}{b}}{\frac{d}{d}}$ & $\frac{\frac{\hbar}{0}}{\frac{0}{0}}$ & $\frac{\pi}{N}$ & $\frac{\hbar}{\frac{\infty}{\pi}}$ \\
\hline 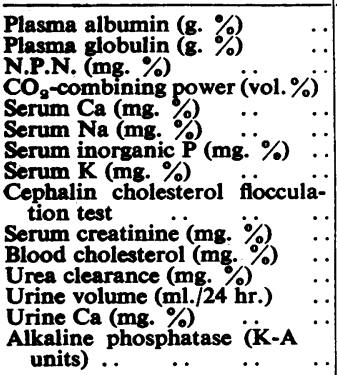 & $\begin{array}{c}4 \cdot 35 \\
2 \cdot 6 \\
128 \\
39 \\
12 \cdot 1 \\
2 \cdot 8 \\
\\
\text { Neg. }\end{array}$ & $\begin{array}{r}5 \\
295\end{array}$ & $\begin{array}{c}36 \\
9 \cdot 9 \\
300 \\
5 \cdot 2 \\
165\end{array}$ & $\begin{array}{l}1,180 \\
10.5\end{array}$ & $\begin{array}{l}9 \cdot 0 \\
5 \cdot 2\end{array}$ & $8 \cdot 6$ & $\begin{array}{l}9 \cdot 7 \\
5 \cdot 3\end{array}$ & 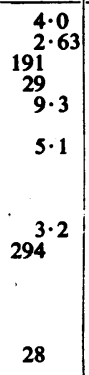 & $\begin{array}{c}9 \cdot 3 \\
\\
\\
1,420 \\
7 \cdot 5\end{array}$ & $\begin{array}{c}1,060 \\
8 \cdot 1\end{array}$ & $\begin{array}{l}9 \cdot 0 \\
4 \cdot 8\end{array}$ & $\begin{array}{c}3 \cdot 31 \\
2 \cdot 26 \\
182 \\
63\end{array}$ & 34 & 48 & 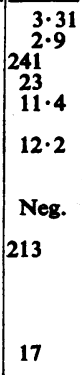 \\
\hline
\end{tabular}

(From May 31 to June 9, 1954, on low Ca diet 0.16 g./24 hours.) 
renal calcification along with the clinical, radiological and biochemical findings exclude the diagnosis in this patient.

Treatment in this case could only be symptomatic in view of the degree of renal failure. The anaemia of uraemia is notoriously resistant to treatment, and the only helpful measure here was transfusion, intravenous iron having no effect.

Alkali was given as 30 grains potassium citrate four times daily from June 26 until the patient left hospital. This was the maximum dose the patient could tolerate and it caused the $\mathrm{CO}_{2}$-combining power to rise to $63 \mathrm{vol}$. \% on June 28 . However, the figure had fallen to $34 \mathrm{vol} . \%$ by June 30 . This change presumably reflected variations in the remaining renal function as other factors were relatively constant. Alkali could hardly be expected to have a sustained effect here as the biochemical findings showed glomerular as well as tubular failure. In this condition the acidosis is due not only to loss of base following failure of ammonia synthesis as in pure tubular lesions, but also to accumulation of acid radicals. It is important to try and distinguish the two types of underlying renal lesion, as the prognosis is reasonably good in some pure tubular lesions if alkali and vitamin $\mathbf{D}$ are given. Massive doses of the latter have been suggested as a means of increasing calcium absorption from the gut, lessening the negative calcium balance and reversing the skeletal changes in hyperparathyroidism secondary to renal acidosis without glomerular insufficiency, the rationale being the lowered gastric acidity and poor calcium absorption in acidosis described by Browne and Vineberg in 1932. It would have been of little avail in the present case. The biochemical findings on her second visit to hospital showed severe uraemia from which she died some weeks later.

\section{Summary}

A case of 'renal rickets' due to probable congenital hypoplasia of the kidneys and secondary hyperparathyroidism is reported. The clinical, radiological and biochemical features are discussed in relation to diagnosis and treatment.

I wish to thank Dr. James K. Slater of Edinburgh Royal Infirmary for permission to publish this case, also the staff of the Radiological and Biochemical Departments for valuable cooperation.

\section{ReFERences}

Albright, F. and Reifenstein, E. C. (1948). The Parathyroid Glands and Metabolic Bone Disease. Baltimore.

Drake, T. G. and Sulkowitch, H. W. (1937). Bull. Johns Hopk. Hosp., 60, 377 .

Browne, J. '̈. L. and Vineberg, A. M. (1932). J. Physiol. (Lond.), 75,345 .

Elis, A. and Evans, H. (1933). Owart. J. Med., n.s. 2, 231.

Fanconi, G. (1936), Jb. Kinderheilk. 147, 299.

Gilmour, J. R (1947). The Parathyroid Glands and Skeleton in Renal Disease, p. 59. London.

MoCune, D. J., Mason, H. H. and Clarke, H. T. (1943). Amer. J. Dis. Child., 65, 81.

Strock, M. S. (1941). New Engl. J. Med., 224, 1019. 